\title{
Robotic surgery in gynecologic oncology: evolution of a new surgical paradigm
}

\author{
John F. Boggess
}

Received: 8 November 2006/ Accepted: 8 January 2007/Published online: 28 February 2007

(C) Springer London 2007

\begin{abstract}
Robotic surgical platforms were first developed with telesurgery in mind. Conceptualized by NASA and the military to provide surgical expertise to remote locations, some telesurgical success has been documented, but progress has been held back by communication bandwidth limitations. Telepresence surgery, where the surgeon is in proximity to the patient but is provided with an ergonomic console equipped with three-dimensional vision and autonomous control of wristed laparoscopic surgical instruments and energy sources, has shown efficacy first in cardiac and then urologic cancer surgery. Interest is currently focused on the application of this technology in the field of gynecology, with techniques being described to perform simple hysterectomy, myomectomy, tubal anastomosis, and pelvic reconstruction procedures. This article will review the application of robotic- and computer-assisted surgery in the specialty of gynecologic oncology.
\end{abstract}

Keywords Gynecology - Gynecologic oncology ·

Minimally invasive surgery · Oncology · Surgical robotics · Surgery

\section{Introduction}

Approximately 85,000 women are diagnosed in the United States each year with gynecologic malignancies. The three most common cancers affecting these women are uterine, ovarian and cervical cancer. Treatment often includes

\section{J. F. Boggess ( $\square)$}

Department of Gynecologic Oncology,

University of North Carolina at Chapel Hill,

4014 Old Clinic Building, Chapel Hill, NC 27599-7570, USA

e-mail: jboggess@med.unc.edu major abdominal surgery to remove the primary cancer usually a total hysterectomy - combined with diagnostic assessment of regional lymph nodes to exclude the presence of metastatic disease. Adjuvant treatment with chemotherapy and radiation therapy is then prescribed based upon surgical staging. Gynecologic oncology patients are often elderly and have medical co-morbidities such as obesity, hypertension, diabetes and cardiovascular disease, which increase their surgical risk. Efforts to reduce surgical morbidity are needed to improve outcomes in this patient population.

The most significant advancement in reducing surgical morbidity in gynecologic oncology over the last 15 years has been an increased application of minimally invasive surgical (MIS) techniques for performing simple hysterectomy, radical hysterectomy, and pelvic and para-aortic lymph node dissection. Feasibility studies have shown safety and efficacy, and limited series have shown oncologic outcomes equivalent to those measured in both overall and progression-free survival [1]. Patients treated laparoscopically have been shown to experience less intraoperative blood loss, less post-operative pain, and shorter hospital stays than those treated by more traditional approaches [2]. Despite these patient advantages, MIS for gynecologic malignancy is still the exception and not the rule. Naumen et al. surveyed members of the Society of Gynecologic Oncologists to evaluate treatment patterns for endometrial cancer. They observed that while $49 \%$ of gynecologic oncologists that responded to the survey stated that they used laparoscopy to stage endometrial cancer, less than $8 \%$ laparoscopically staged at least half of their patients. Some reasons cited for this limited use of laparoscopy in gynecologic oncology include longer operative times, a steep learning curve to adopt minimally invasive techniques, lack of training for surgeons who have already 
completed formal medical training, and a perceived inferiority of some of the procedures. Robotic-assisted surgical platforms may overcome many of the shortcomings of laparoscopy while preserving the patient benefits.

Given that we are describing a new era of surgical technology and techniques, it is important to understand some terms. An excellent review written by David Camarillo et al. summarizes the evolution of robotics in surgery. Dr. Camarillo [3] points out that the term "robot" was originally coined by playwright Karel Capek in his satirical drama Rossum's Universal Robots, written in 1920 . He derived the word robot from the Czech rabota, meaning slave labor. In the play, machines were made to do mundane work so that people would be free to pursue more creative interests. In the end, the machines became smarter than their "masters", ultimately leading to the destruction of the latter. Today's surgical "robots" are not by strict definition robots in that they do not perform independent tasks and do not exhibit artificial intelligence. Instead, it might be better to refer to this technology as computerassisted surgery. Nonetheless, robotic surgery has already been accepted as a surrogate descriptor for computer-assisted surgery and will be used throughout this text.

This article chronicles the evolution of one robotic surgery program in gynecologic oncology with emphasis on issues pertaining to application, procedure development, training, research, and cost. This article is not intended to be a review, mainly because we are at the beginning of an era of robotics and not at the end or even in the middle. This article is also not intended to be a comprehensive description of techniques as such articles will be forthcoming; it focuses instead on the implications of developing a robotic surgical program and hints at the promise this technology holds for improving the quality of life of gynecology cancer patients by reducing surgical morbidity.

\section{Developing a surgical robotics program in gynecologic oncology}

\section{Surgical robots}

There is currently only one robotic surgical platform commercially available and FDA approved for performing gynecologic oncology procedures - the daVinci surgical system (Intuitive Surgical Inc, Sunnyvale, Calif.). Introduced in 1999, the daVinci is comprised of three components: the patient side surgical cart, the vision system, and the surgeon console (Fig. 1). The surgical cart is composed of three to four arms for controlling a 12-mm threedimensional (3D) camera and two to three surgical instruments. The surgical cart is "docked" to proprietary laparscopic trocars placed in the patient's abdomen. The

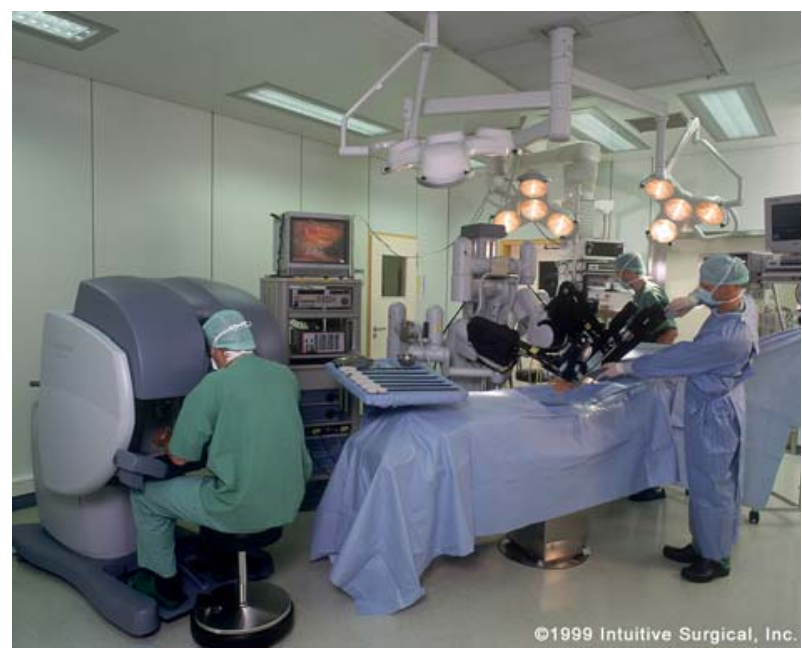

Fig. 1 daVinci Surgical System (photo courtesy of Intuitive Surgical, Sunnyvale, Calif.)

video signal from each of two charged coupled device (CCD) cameras (Fig. 2) is then processed independently by the vision cart, delivered to the surgeon console, and displayed on two separate monitors. These two monitors are focused for the surgeon at the console and viewed as the "right" and "left" eye to reconstruct a 3D immersive view of the surgical field. The robotic instruments are "wristed", thereby providing $7 d f$ compared with the $4 d f$ with traditional laparoscopy (Fig. 3). The robotic instruments are controlled by the primary surgeon, who sits away from the patient at the surgical console via two "masters". The surgeon's movements are translated in real-time to the robotic instruments placed within the patient and are scaled and processed to reduce tremor and to enhance precision. The surgical masters are placed inline with the surgeon's field of vision so as to restore a more intuitive eye-hand relationship than that found with traditional laparoscopy. Also at the surgical console are foot-controlled clutches for

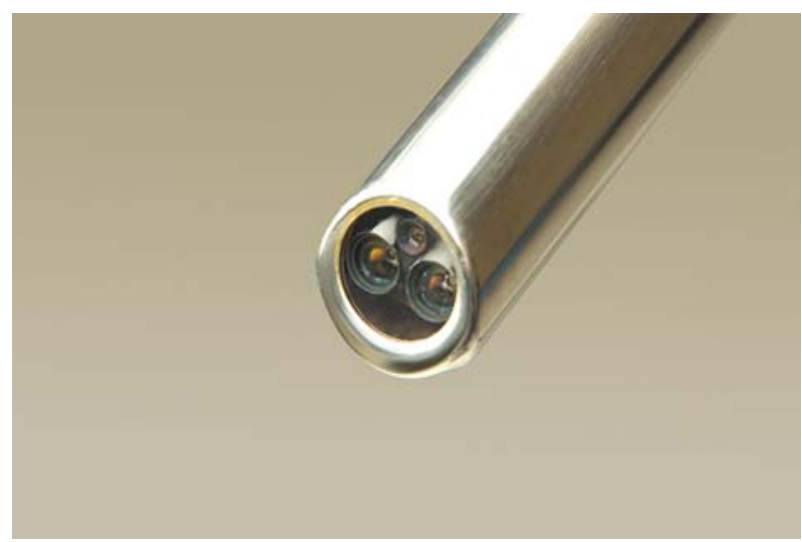

Fig. 2 InSight 3-D Camera System (photo courtesy of Intuitive Surgical) 


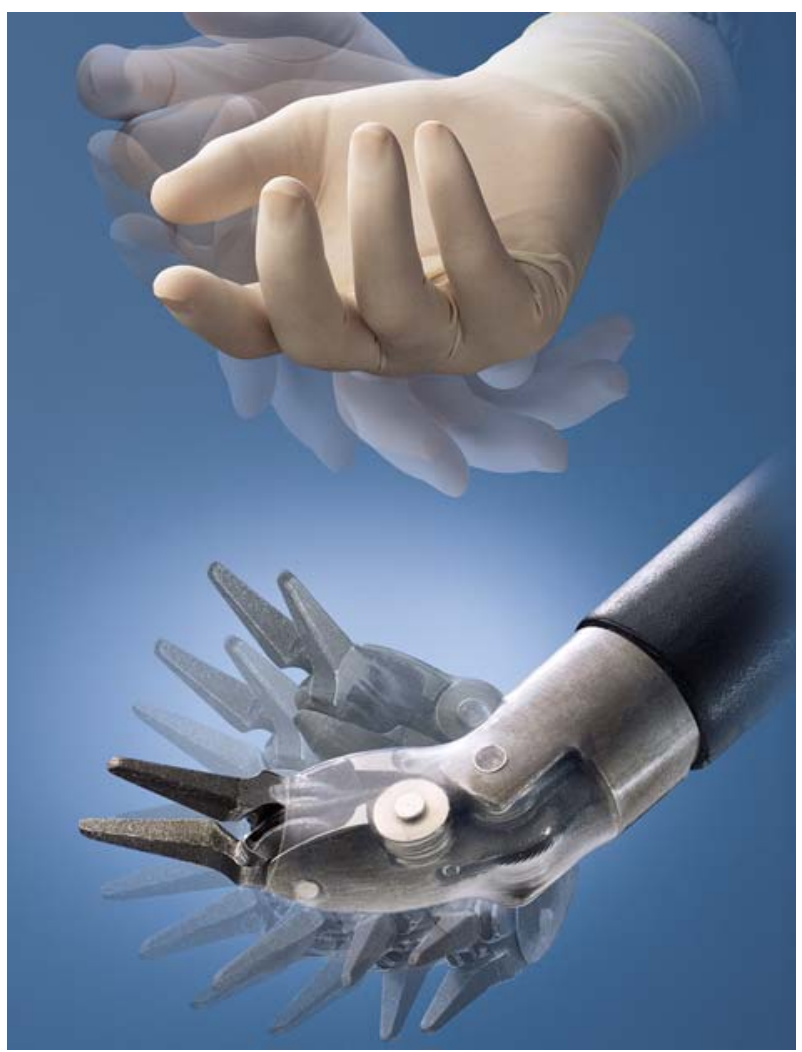

Fig. 3 EndoWrist robotic instrument (photo courtesy of Intuitive Surgical)

camera movement, master position, and activation of the energy sources. The primary surgeon works autonomously at the console remote from the patient, while a surgical assist remains scrubbed at the patient's side to assist with retraction, suction/irrigation, and passing of needles and sponges.

The food and drug administration approved the use of daVinci for performing gynecologic procedures in April 2005 based largely upon preliminary data provided by the University of Michigan on robotic-assisted uterine myomectomies and hysterectomies carried out at their hospital [4]. At that time, there were no published descriptions of techniques for performing robotic procedures on gynecologic oncology patients.

\section{Robotic surgical training}

Following the installation of a daVinci surgical system in February 2005 at the University of North Carolina, the author set out to assess the feasibility and efficacy of robotic assistance in performing gynecologic oncology procedures. Based on over 7 years of experience performing advanced laparoscopic procedures for the treatment of gynecologic malignancies, this author sought to translate these techniques to the robotic platform with the goal of establishing standardized, reproducible robotic techniques for performing simple and radical hysterectomy and pelvic and para-aortic lymph node dissection in the context of treating cervical, endometrial and early ovarian cancer. Preparation included an on-line orientation to the daVinci system, a 2-h dry lab spent suturing and performing simple dexterity skills with the robotic surgical system, and a formal porcine training lab provided at East Carolina University. The skills emphasized during this course included a comprehensive understanding of the entire system, including set-up, draping, and equipment calibration. A retroperitoneal dissection, nephrectomy, suturing, knot tying, and anastomosis was then carried out on a live porcine model in order to assimilate the robotic system in a setting that most approximated the skills required to perform hysterectomy and node dissection. This training was completed within 1 week of the first planned robotic-assisted operative case, a simple hysterectomy and bilateral salpingo-oophorectomy for a $10-\mathrm{cm}$ dermoid ovarian cyst. Based upon success with this first application, the daVinci surgical system has been used by the author since May 2005 to successfully perform nearly 150 robotic cases, with over $80 \%$ being performed for the treatment of gynecologic malignancy.

\section{Procedure development}

While there is reference to the staging of gynecologic malignancy in an early series of robotic-assisted hysterectomy cases, there were no published or even un-published descriptions of this technique or standardized approaches to performing robotic-assisted hysterectomy or lymph node dissection when we began our program [5]. We therefore, translated an already familiar technique of total laparoscopic hysterectomy described by Koh et al. [6] in 1998 for performing simple hysterectomy, which incorporates a Zumi uterine manipulator, $\mathrm{KOH}$ colpotomizer rings, and a pneumo-occluder balloon to the daVinci (Cooper Surgical, Trumbull, Conn.). The $\mathrm{KOH}$ system facilitates para-cervical dissection when performing simple hysterectomy and has been reviewed in many clinical series. For radical hysterectomy, the uterine manipulator was replaced with a rectal dilator placed in the vagina and used as a stent to delineate the vaginal wall for parametrial dissection and colpotomy. Radical hysterectomy was performed in the same manner as when performing the surgery abdominally. Trans-peritoneal lymph node dissection was performed following the International Federation of Gynecology and Obstetrics (FIGO) guidelines for the staging of endometrial, cervical, and ovarian cancer. All surgical techniques adhered to the same approaches taken with traditional laparotomy with respect to anatomic planes and surgical margins, with the exception that clamps and sutures were 
replaced with bipolar and monopolar energy sources for vessel sealing. A meticulous prospective collection of operative times broken down into components, including the induction of anesthesia and patient positioning, trocar placement, and the docking of the robotic surgical system; in addition, the individual components of a hysterectomy and lymph node dissection were performed for each case in order to track progress and critically assess areas for refinement of technique. Nearly all procedures were video recorded for review by the primary surgeon to facilitate critical assessment of the technique and streamline the flow of the operation.

Table 1 summarizes the procedures performed from May 2005 through October 2006 by the gynecologic oncology team at the University of North Carolina at Chapel Hill. Specific surgical outcomes will be reported in subsequent publications, but many observations can be mentioned here. First, it must be made clear that we emphasized the robotic application for simple hysterectomy, radical hysterectomy, and pelvic and para-aortic lymph node dissection in the context of cervical and endometrial cancer. Only limited experience exists thus far for staging early ovarian cancer. This is primarily due to the design of the current robotic surgical system and the range of motion of the instruments. In order for daVinci to reach the upper abdomen, as is necessary in high paraaortic lymph node dissection, the ports need to be placed very high on the abdomen. There is a limit as to how high the ports can be placed and still operate in the deep pelvis due to the range of motion of the instruments. When developing these procedures, we were committed to developing a single docking approach that emphasized simplicity and reproducibility. While in some patients the high para-aortics can be reached with the system still providing enough range of motion to complete hysterectomy and pelvic node dissection, this is not generally true.

Table 1 Gynecologic oncology robotic procedures performed between May 2005 and October 2006

\begin{tabular}{ll}
\hline Procedure & $\begin{array}{l}\text { Number of } \\
\text { operations }\end{array}$ \\
\hline Simple hysterectomy & 33 \\
Simple hysterectomy with pelvic and para-aortic & 51 \\
$\quad$ nodes & 39 \\
Radical hysterectomy with pelvic nodes & 2 \\
Simple hysterectomy with ovarian cancer & \\
$\quad$ staging & 5 \\
Oophorectomy & 6 \\
Ovarian cystectomy in pregnancy & 6 \\
Other & 142 \\
Total & \\
\hline
\end{tabular}

This limitation in design has been partially overcome with the newest generation of daVinci and accounts for the successful completion of the ovarian staging procedures listed in our series. However, increasing the ease and ability to perform true four quadrant surgery will need to be emphasized in future robotic surgical system design. What is striking, however, is that the current platform, while not originally designed to perform gynecologic procedures, allowed us to treat consecutive patients with both cervical and endometrial cancer without the need for conversion to laparotomy. This observation alone provides the clearest glimpse into the potential advantages of robotic-assisted surgery, when one considers that this series represents not only a learning curve, but the initial development of technique.

\section{Clinical experience}

Several reports have been published in the last 2 years that describe robotic-assisted simple hysterectomy in terms of technique and experiences [7-10]. While these reports demonstrate feasibility, they are limited in numbers, and their results will need to be validated with larger studies. Reynolds et al. [11] published their initial experience staging seven patients with gynecologic malignancy, four patients with endometrial cancer, two with ovarian cancer, and one with fallopian tube cancer. The authors were able to complete staging in all patients without conversion to laparotomy, however, they were required to re-dock the system in order to perform high para-aortics. They recovered an average of 15 lymph nodes. None of their patients required blood transfusion, and no significant complications were experienced. Marchal et al. [12] reported in 2005 on their initial experience with 30 patients. Twelve of their patients had robotic-assisted surgery for gynecologic malignancy (seven uterine and five cervix). While details on the extent of the surgery are not available, these researchers reported performing Type II radical hysterectomy for some patients, and pelvic node dissection for nine patients in total. They concluded that robotic-assisted surgery was feasible and more ergonomic than laparoscopy.

We reported our initial experience with robotic-assisted radical hysterectomy with bilateral pelvic lymphnode dissection at the Society of Gynecologic Oncologists meeting in March 2006. In that report, we compared the surgical and pathologic outcomes of 13 consecutive robotic-assisted Type III radical hysterectomies with 48 historic abdominal radical hysterectomies. All of the robotic procedures were completed successfully without any conversion to laparotomy. There were significantly more lymph nodes recovered robotically than abdominally (33 vs. 22; $P=0.001$ ), no increase in operative time (median 242 vs. 
$240 \mathrm{~min}$ ), less blood loss (100 vs. $400 \mathrm{cc}$ ), and no blood transfusions administered to the robotic-assisted surgery patients compared with blood transfusions to $8 \%$ of the abdominal controls. Furthermore, all of the robotic-treated patients were discharged within $24 \mathrm{~h}$, and none required intra-venous pain medication compared with an average of three hospital days for the abdominal group.

During a session on laparoscopic lymph node dissection at the American College of Surgeons, we reported unpublished data on endometrial staging in which we compared a series of 43 patients staged robotically versus 101 patients staged laparoscopically. None of the robotic patients were converted to laparotomy versus $3 \%$ for the laparoscopy group; in addition, significantly more nodes were retrieved (30 vs. $23, P=0.004$ ), less blood was lost (63 vs. $142 \mathrm{cc}$; $P=0.0001)$, a shorter operative time was required (163 vs. $213 \mathrm{~min} ; P=0.002)$, and a shorter hospitalization period was necessary ( 1 vs. 1.2 days; $P=0.04$ ) with the robotic cohort than with the laparoscopy cohort. The most significant point may be that we were able to perform comprehensive staging on larger women (BMI 33 vs. 29; $P=0.008$ ). These data suggest that robotic assistance improves upon our already established laparoscopic approach to the treatment of endometrial cancer.

As our comfort level with the daVinci surgical system grew through experience, we expanded our indications, as can be observed in Table 1. In addition, our residents and fellows in training are routinely involved in all procedures and are being trained at the console to perform first the hysterectomy and then pelvic node dissection. We have found that training residents in this setting is safe, and our early experience would suggest that their learning curve is faster than with laparoscopy due to the intuitive nature of the system. The two gynecologic oncology fellows that have been exposed to robotics and subsequently graduated are both actively using robotics in their respective practices, thereby demonstrating that our investment in their learning robotics as part of their oncology training was worthwhile. It will be important for training programs to balance the need for the teachers to learn in order to then teach the students. It is this author's belief that robotics enhances the training of anatomy and technique by emphasizing precision. Issues such as certification of graduating trainees will need to be standardized as training programs become more widespread. The Society of Gynecologic Oncologists as well as the American College of Obstetricians and Gynecologists should take a proactive role in standardizing training and certification.

Costs and institutional dynamics

Cost analysis is an important and difficult task when evaluating any new technology. When establishing a robotics program, there is clearly a significant fixed cost associated with the purchase of a robotic surgical system. The first cost includes the purchase price of the robotic system, which ranges between $\$ 1,000,000$ and $\$ 1,500,000$ and requires a $10 \%$ annual maintenance fee for repair and service as well as software upgrades to the system. The second cost is the procedure disposable cost, which includes the robotic instruments (\$200/use), drapes to maintain sterility of the system, and a few other accessories and ports required to perform the surgery that are specific to robotics. The third cost is the cost of training new personnel and initial delays in setup time and procedure time during the learning curve. The fourth cost is the expense of training and/or proctoring until the surgeon is certified. This cost is highly variable. depending upon institutional policies and the availability and cost of trained proctors.

Although we have not yet performed a detailed cost analysis of our robotics program, some observations can be shared. The fixed cost depends greatly upon the number of cases being performed over the amortized life span of the robotic system. For example, if a $\$ 1,500,000$ robotic system with a $10 \%$ per year service contract is amortized over 7 years, then the fixed cost for a single surgical system is approximately $\$ 365,000$ per year. If two cases are performed per day, 5 days/week for a total of 520 cases annually, the fixed cost per case is approximately $\$ 700$. This cost would increase dramatically if the number of cases performed is substantially less. The disposable costs for our procedures are approximately $\$ 1000$ (three to four robotic instruments, drapes, one to two disposable trocars). This cost is typically less than the disposable costs for comparable cases performed laparoscopically at our institution. The added operative costs are offset by a shortened hospital stay and a decreased need for post-operative pain medication compared with open abdominal surgery. The cost offset becomes less when comparing robotic to laparoscopic techniques. We have observed that the operating room setup for robotic cases is approximately $15 \mathrm{~min}$ longer than for laparoscopy. Over time, our operative times have become substantially less compared with laparoscopy and comparable to laparotomy. Docking the system quickly becomes trivial in terms of time, and it takes an experienced team less than $5 \mathrm{~min}$. In summary, when analyzing cost, it is important to maximize utilization, to strive for efficiency, both in preparation for and during the operation, and to balance increased procedure costs with post-operative care plans that yield early discharge from the hospital.

An approximate $10 \%$ return on the investment has been estimated for hospitals that have initiated urology robotics programs; this is due largely to changes in regional referral patterns of patients for specialized services, an increase in ancillary charges, and shorter patient stays. Whether a similar return on the investment will be observed in 
gynecologic oncology will depend upon whether robotics allows more surgeons to adapt minimally invasive surgical procedures to their practice. As more and more systems are being installed, the "novelty" of having a robot will disappear, and regional growth of programs will stabilize. Robotics programs will need to pay for themselves on a per case basis. Furthermore, competition for access to robotic systems is already a major problem for many centers as multiple surgeons within a department and between departments are sharing an expensive and limited resource. We currently operate two robotic systems at the University of North Carolina; these systems support programs in Urology, Gynecology, gynecologic oncology, Pediatric Surgery, and General Surgery. The coordination of scheduling and cooperation between surgeons has become increasingly critical in maintaining efficient utilization of the systems while serving the needs of each individual surgeon's growing program. Ultimately, widespread growth of robotics will require that costs come down. This will most likely happen when competitors enter the market, and indications are validated and expanded across surgical disciplines, thus increasing the demand based upon superior patient outcomes. Surgical robotics will have longevity only if demonstrated to be "better medicine" not better business.

\section{Summary}

A great deal of progress has occurred in the field of robotic surgery and gynecologic oncology over the last 2 years, and this has generated a great deal of interest within our specialty. Despite this development, this technology and its application in gynecologic oncology are still in their infancy. We and others have established comprehensive robotic programs incorporating routine clinical application in an effort to develop standardized procedures and evaluate efficacy. We have presented early clinical outcomes at national meetings and the publication of larger case series is soon to follow. Furthermore, we have developed detailed procedure manuals with video documentation of techniques so that surgeons new to robotics have the benefit of a standardized approach. A few of us with significant experience have established training programs that allow surgeons to come and learn from our experience and then observe live robotic cancer cases. The potential for robotic and computer-assisted surgical devices to revolutionize our specialty by nearly eliminating post-operative morbidity while preserving radicality and the tenets of oncology surgery for a large majority of the patients that we treat seems to be right around the corner. However, it is our responsibility as a specialty caring for women with cancer to standardize the technique and subject our outcomes to peer review before we can recognize this development as a new standard of care. In order to do this, it is essential to establish a national registry of cases for the research, expansion, and support of training centers of excellence and partnering with industry in order to develop tomorrow's tools.

The brilliance of this technology is simple: by restoring dexterity, 3D vision, and autonomy to the abdominal surgeon while leveraging the patient outcome advantages that come from minimally invasive techniques, better surgical outcomes will be achieved. In addition, as has been seen in Urology, we should experience shallower learning curves and greater generalizability to more practitioners previously discouraged by the limitations of standard laparoscopy. Many naive surgeons have described robotic-assisted surgery as a "fancy laparoscope". On the contrary, laparoscopy was an early evolutionary step toward robotics, and we are experiencing that evolution now. If the success witnessed in Urology and the treatment of prostate cancer is an indicator for our specialty, and I believe it should be, robotic and computer-assisted technology will allow more surgeons to perform a greater diversity of complex minimally invasive procedures. As costs are contained, even simple, more common procedures such as hysterectomy will be performed with less morbidity to the patient than even laparoscopy. It will truly be an exciting decade as we witness the evolution of a new surgical paradigm in gynecologic oncology.

\section{References}

1. Magrina JF, Weaver AL (2004) Laparoscopic treatment of endometrial cancer: five-year recurrence and survival rates. Eur J Gynaecol Oncol 25(4):439-41

2. Abaid L, Boggess J (2005) Current applications of laparoscopy in gynecologic oncology: a literature review. Women's Oncol Rev 5(4):193-201

3. Camarillo DB, Krummel TM et al. (2004) Robotic technology in surgery: past, present, and future. Am J Surg 188[Suppl 4A]:2S$15 \mathrm{~S}$

4. Advincula AP, Song A et al. (2004) Preliminary experience with robot-assisted laparoscopic myomectomy. J Am Assoc Gynecol Laparosc 11(4):511-518

5. Diaz-Arrastia C, Jurnalov C et al. (2002) Laparoscopic hysterectomy using a computer-enhanced surgical robot. Surg Endosc 16(9):1271-1273

6. Koh CH (1998) A new technique and system for simplifying total laparoscopic hysterectomy. J Am Assoc Gynecol Laparosc 5(2):187-192

7. Beste TM, Nelson KH et al (2005) Total laparoscopic hysterectomy utilizing a robotic surgical system. J Soc Laparoendosc Surg 9(1):13-15

8. Reynolds RK, Advincula AP (2006) Robot-assisted laparoscopic hysterectomy: technique and initial experience. Am J Surg 191(4):555-560 
9. Fiorentino RP, Zepeda MA, Goldstein BH (2006) Pilot study assessing robotic laparoscopic hysterectomy and patient outcomes. J Minim Invasive Gynecol 13(1):60-63

10. Advincula AP, Reynolds RK (2005) The use of robot-assisted laparoscopic hysterectomy in the patient with a scarred or obliterated anterior cul-de-sac. J Soc Laparoendosc Surg 9(3):287-291
11. Reynolds RK, Burke WM, Advincula AP (2005) Preliminary experience with robot-assisted laparoscopic staging of gynecologic malignancies. J Soc Laparoendosc Surg 9(2):149-158

12. Marchal F, Rauch P, Vandromme J (2005) Telerobotic-assisted laparoscopic hysterectomy for benign and oncologic pathologies: initial clinical experience with 30 patients. Surg Endosc 19(6):826-831 